\title{
28. Sur la théorie des espaces à hyperconnexion euclidienne, II.*
}

Par Kentaro YANO.

Institut Mathématique, Université Impériale de Tokyo.

(Comm. by S. KAKEYA, M.I.A., March 12, 1945.)

§4. Les tenseurs de courbure.

Le tenseur de courbure $R_{\cdot j k h}^{i}$ de Riemann-Christoffel de $V_{n}$ est donné par

$$
R_{\cdot j k h}^{i}=\left\{\begin{array}{c}
i \\
j k
\end{array}\right\}_{, h}-\left\{\begin{array}{c}
i \\
j h
\end{array}\right\}_{, k}+\left\{\begin{array}{c}
a \\
j k
\end{array}\right\}\left\{\begin{array}{c}
i \\
a h
\end{array}\right\}-\left\{\begin{array}{c}
a \\
j h
\end{array}\right\}\left\{\begin{array}{c}
i \\
a k
\end{array}\right\} .
$$

Il apparait dans la formule de Ricci:

$$
v_{; k ; h}^{i}-v_{; k ; k}^{i}=v^{j} R_{\cdot j k h}^{i},
$$

et satifait aux relations algébriques :

$$
\begin{array}{ll}
R_{\cdot j k h}^{i}=-R_{\cdot j h k}^{i}, & R_{\cdot j k h}^{i}+R_{\circ k h j}^{i}+R_{\circ h j k}^{i}=0, \\
R_{i j k h}=-R_{j i k h}, & R_{i j k h}=R_{k h i j},
\end{array}
$$

où l'on a posé $R_{i j k h}=g_{i a} R_{\cdot j k h}^{a}$,

De plus, il satisfait à l'identité bien connue de Bianchi:

$$
R_{\cdot j k h ; l}^{i}+R_{\cdot j h i ; k}^{i}+R_{\cdot j l k ; \hbar}^{i}=0,
$$

d'où

$$
\left(R_{\bullet k}^{i}-\frac{1}{2} R \delta_{k}^{i}\right)_{; i}=0
$$

où nous avons posé $R_{\bullet k}^{i}=g^{i j} R_{j k}$ et $R_{j k}=R_{\cdot j k i}^{i}, R_{j k}$ étant le tenseur de Ricci qui est symétrique par rapport aux deux indices inférieurs.

Cela étant, nous allons considérer le tenseur de courbure de $\boldsymbol{E}_{m}$. Pour cela, calculons $V_{; k ; h}^{\lambda}-V_{; h ; k}^{\lambda}$, on trouve, par un'calcul facile,

$$
V_{; k ; h}^{\lambda}-V_{; h ; k}^{\lambda}=V^{\mu} K_{. \mu k h}^{\lambda},
$$

où

$$
K_{\bullet \mu k h}^{\lambda}=\Gamma_{\mu k, h}^{\lambda}-\Gamma_{\mu h, k}^{\lambda}+\Gamma_{\mu, k}^{\alpha} \Gamma_{\alpha h}^{\lambda}-\Gamma_{\mu h}^{a} \Gamma_{\alpha k}^{\lambda} .
$$

Les composantes du tenseur de courbure ainsi définies satisfont aux relations algébriques

$$
K_{. \mu k h}^{\lambda}=-K_{\cdot \mu h k}^{\lambda}, \quad K_{\lambda \mu k h}=-K_{\mu \lambda k h},
$$

la deuxième étant une conséquence de l'identité:

$$
0=G_{\lambda \mu ; k ; h}-G_{\lambda \mu ; h ; k}=-G_{\alpha \mu} K_{\cdot \lambda k h}^{\alpha}-G_{\lambda \alpha} K_{\cdot \mu k h}^{\alpha} \text {. }
$$

Calculons cette fois $B_{j ; k ; h}^{\cdot \lambda}-B_{j ; h ; k}^{\cdot \lambda}$, alors on trouve

$$
B_{j ; k ; h}^{\cdot \lambda}-B_{j ; h ; k}^{\cdot \lambda}=B_{j}^{\cdot \mu} K_{\cdot \mu k h}^{\lambda}-B_{i}^{\cdot \lambda} R_{\cdot j k h}^{i} \text {. }
$$

* La dépense de cette recherche fut réglée par le frais du Ministère de l'Tnstruction. Publique pour les recherches scientifiques.

La première partie de cette Note fut publiée dans ce Proc., 21 (1945), 156-163. 
En substituant (2.8) dans cette équation et en tenant compte de (2.15), on obtient

$$
\begin{aligned}
\left(H_{j k ; h}^{\cdot{ }^{P}}-H_{j h ; k}^{\cdot P}\right) B_{P}^{\cdot \lambda} & -B_{i}^{\cdot \lambda}\left(H_{j k}^{\cdot \bullet P} H_{\cdot h P}^{i}-H_{j h}^{\cdot{ }^{P}} H_{\cdot k P}^{i}\right) \\
& =B_{j}^{\cdot \mu} K_{\cdot \mu k h}^{\lambda}-B_{i}^{\cdot \lambda} R_{\cdot j k h}^{i}
\end{aligned}
$$

d'où on tire

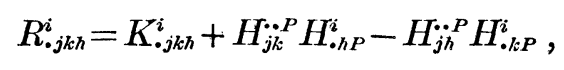

et

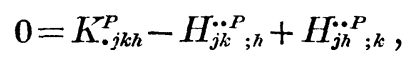

où nous avons posé

$$
K_{\cdot{ }^{\prime} j k h}^{i}=B_{{ }_{\lambda} \lambda}^{i} B_{j}^{\cdot \mu} K_{\cdot \mu k h}^{\lambda} \quad \text { et } \quad K_{\bullet j k h}^{P}=B_{\bullet_{\lambda}}^{P} B_{j}^{{ }^{\mu}} K_{\cdot \mu k h}^{\lambda}
$$

La deuxième identité $d \epsilon$ (4.3) et l'équation (4.11) nous donnent

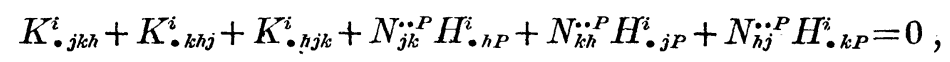

où nous avons posé

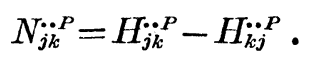

D'aute part, l'équation (4.1.2) nous donne

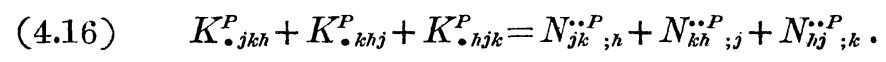

De plus, si l'on pose $K_{i j k h}=g_{i a} K_{\cdot j k h}^{a}$, on obtient, de (4.11),

$$
K_{i j k h}=-K_{j i k h} \text {. }
$$

Cela étant, calculons cette fois $B_{\dot{Q}^{\lambda} ; k ; h}-B_{Q_{Q}{ }^{\lambda} ; ; k}$, alors on trouve

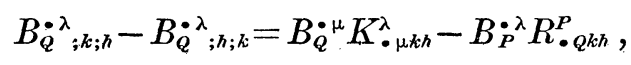

où nous avonṣ posé

$$
R_{\cdot Q^{k h}}^{P}=\Gamma_{Q^{k, h}}^{P}-\Gamma_{Q^{h, k}}^{P}+\Gamma_{Q^{k}}^{R} \Gamma_{R_{h}}^{P}-\Gamma_{Q^{h}}^{R} \Gamma_{k k}^{P}
$$

Ce tenseur de courbure de $E_{m-n}$ satisfait aux relations algébriques

$$
R_{\cdot Q k h}^{P}=-R_{\cdot Q \hbar k}^{P}, \quad R_{P Q k h}=-R_{Q P k h},
$$

la deuxième étant une conséquence de

$$
0=g_{P Q ; k ; h}-g_{P Q ; \hbar ; k}=-g_{R Q} R_{\cdot P k h}^{R}-g_{P R} R_{\cdot Q k h}^{R} .
$$

Or, en substituant (2.15) dans (4.18), et en tenant compte de (2.8), on trouve

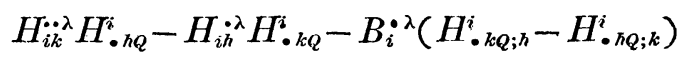

$$
\begin{aligned}
& =B_{Q}^{\cdot{ }^{\mu}} K_{\bullet \mu k h}^{\lambda}-B_{P}^{{ }^{\lambda}} R_{\bullet Q^{k h}}^{P},
\end{aligned}
$$

d'où on tire

$$
H_{\bullet k Q ; h}^{i}-H_{\bullet k Q: k}^{i}=-B_{\cdot \lambda}^{i} B_{Q}^{{ }^{\mu}} K_{\cdot \mu k h}^{\lambda},
$$

et

$$
R_{\cdot Q k h}^{P}+H_{i k}^{* P} H_{\cdot{ }^{\prime} Q}^{i}-H_{i \hbar}^{* P} H_{*_{k Q}}^{i}=0 .
$$

Comme nous avons $-B_{{ }_{\lambda}{ }_{\lambda} B_{Q}{ }^{\mu} K_{\bullet \mu k h}^{\lambda}}^{\lambda}=g_{P Q} g^{i j} K_{\cdot j k h}^{P}$, l'équation (4.22) est équivalente à (4.12).

Or, nous avons déjà démontré la formule de Ricci (4.6). 
Appliquons cette formule à un tenseur mixte $V_{; k}^{\lambda}$. Nous aurons, par un calcul facile,

$$
V_{; k ; h ; l}^{\lambda}-V_{; k ; l ; h}^{\lambda}=V^{\mu}{ }_{; k} K_{\bullet \mu h l}^{\lambda}-V^{\lambda}{ }_{i i} R_{\bullet k h l}^{i} .
$$

D'autre part, en dérivant covariantement la formule (4.6), on a

$$
V_{; k ; h ; l}^{\lambda}-V_{; ; ; k ; l}^{\lambda}=V_{; l}^{\mu} K_{\bullet \mu k h}^{\lambda}-V^{\mu} K_{\bullet \mu k h ; l}^{\lambda} \text {. }
$$

On permute les indices $k, h, l$ cycliquement dans (4.24) et dans (4.25), et on compare les équations obtenues, alors en tenant compte de l'identité (4.3), on trouve

d'où

$$
V^{\mu}\left(K_{\bullet \mu k h ; l}^{\lambda}+K_{\bullet \mu h l ; k}^{\lambda}+K_{\bullet \mu l k ; h}^{\lambda}\right)=0
$$

$$
K_{\bullet \mu k h ; l}^{\lambda}+K_{\bullet \mu h l ; k}^{\lambda}+K_{\bullet \mu k ; k}^{\lambda}=0
$$

$V^{\mu}$ étant tout à fait arbitraire. La formule (4.26) nous donne l'identité de Bianchi pour le tenseur de courbure $K_{\bullet_{\mu k h}^{\lambda}}^{\lambda}$.

On peut démontrer, par un procédé analogue, l'identité de Bianchi

$$
R_{\bullet Q k h ; l}^{P}+R_{\cdot Q \hbar l ; k}^{P}+R_{\cdot Q l k ; h}^{P}=0
$$

pour le tenseur de courbure $R_{\bullet}^{P}{ }^{k h \hbar}$.

§5. La détermination du tenseur de courbure $K_{\bullet \mu k h}^{\lambda}$ de $E_{m}$.

Nous avons trouvé trois tenseurs de courbure, celui de $V_{n} R_{\bullet j k h}^{i}$, celui de $E_{m-n} R_{\bullet Q k h}^{P}$ et celui de $E_{m} K_{\bullet \mu l \hbar \bullet}^{\lambda}$ Nous allons montrer, dans ce Chapitre, que les composantes $K_{\bullet \mu k h}^{\lambda}$ du tenseur de courbure de $E_{m}$ pervent se déterminer en termes de $R_{\cdot j k h}^{i}, R_{\bullet Q k h}^{P}$ et $H_{j k}{ }^{P}$, le tenseur qui relie les connexions de $V_{n}$ et $E_{m-n}$ à celle de $E_{m}$.

Des équations (4.10), on obtient, en contractant $B^{j}{ }_{\mu}$,

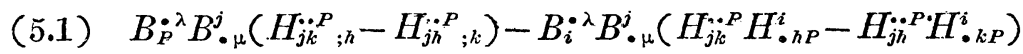

$$
\begin{aligned}
& =B^{j}{ }_{\mu} B_{j}^{* \alpha} K_{\bullet \alpha k h}^{\lambda}-B_{i}^{\cdot \lambda} B^{j}{ }_{\mu \mu} R_{\bullet j k h}^{i} .
\end{aligned}
$$

D'autre part, des équations (4.21), on trouve, en contractant $B_{\cdot \mu}^{Q}$,

$$
\begin{aligned}
& H_{i k}^{i^{\lambda \lambda}} H_{\bullet h \mu}^{i}-H_{i h{ }^{-\lambda}} H_{\bullet k \mu}^{i}-B_{i}^{* \lambda} B_{\bullet \mu}^{Q}\left(H_{\bullet k Q ; h}^{i}-H_{\bullet h q ; k}^{i}\right) \\
& =B_{\cdot \mu}^{Q} B_{Q^{\alpha}}^{{ }^{\alpha}} K_{\cdot \alpha k h}^{\lambda}-B_{P}^{{ }^{\lambda}} B_{\cdot \mu}^{Q} R_{\cdot Q^{k h}}^{P} .
\end{aligned}
$$

En ajoutant (5.1) et (5.2) et remarquant que $B_{\bullet \mu}^{j} B_{j}^{\cdot \alpha}+B_{{ }_{\mu}}^{\alpha} B_{Q}^{{ }^{\alpha}}=\delta_{\mu}^{\alpha}$, on trouve

$$
\begin{aligned}
& K_{\bullet \mu k h}^{\lambda}=B_{i}^{\cdot \lambda} B_{\bullet \mu}^{j} R_{\cdot j k h}^{i}+B_{P}^{{ }^{\lambda}} B_{\cdot \mu}^{Q} R_{\bullet Q^{k h}}^{P}
\end{aligned}
$$

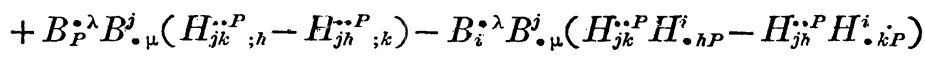

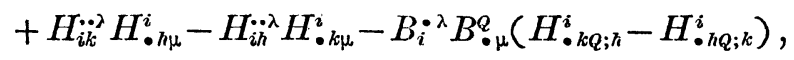

ce qui est une géneralisation d'une iormule donnée par MM. Michal et Botsford.

Le tenseur de courbure $K_{\bullet \mu k h}^{\lambda}$ de $E_{m}$ étant donné par la formule (5.3), nous posons

$$
K_{\mu k}=B_{{ }^{\prime}}^{\lambda} K_{\cdot \mu k h}^{\lambda},
$$


et l'appelons, le tenseur d'Einstein-Mayer. De (5.3) on obtient

(5.5) $K_{\mu k}=B_{\cdot \mu}^{j}\left(R_{j k}-H_{j k}^{\cdot{ }^{P}} H_{\bullet a P}^{a}+H_{j a}^{\cdot P} H_{\bullet k P}^{a}\right)$

$$
-B_{\cdot \mu}^{Q}\left(H_{\bullet k Q ; a}^{a}-H_{\bullet a Q ; k}^{a}\right) \text {. }
$$

Cela étant, posons encore

$$
K=B^{k \mu} K_{\mu k},
$$

alors, on aura, de (5.5),

$$
K=R-H_{\bullet a}^{a \cdot P} H_{\bullet b P}^{b}+H_{\bullet_{b}^{*}}^{a} H_{\bullet a P}^{b},
$$

$K$ étant la courbure scalaire de $E_{m}$.

§6. Les lignes les plus droites relativement à $E_{m}$.

La ligne géodésique de $V_{n}$ est donnée par

$$
\frac{\delta^{2} x^{i}}{d s^{2}}=\frac{d^{2} x^{i}}{d s^{2}}+\left\{\begin{array}{c}
i \\
j k
\end{array}\right\} \frac{d x^{j}}{d s^{\prime}} \frac{d x^{k}}{d s}=0 .
$$

Donc, le vecteur $\frac{d x^{i}}{d s}$ tangent à la courbe se déplace parallèlement à luimême; si l'on déplace, le long de cette courbe, le vecteur $B_{i}^{* \lambda} \frac{d x^{i}}{d s}$ de $E_{m}$ tangent à la courhe, on trouve

$$
\frac{\delta}{d s}\left(B_{i}^{\cdot \lambda} \frac{d x^{i}}{d s}\right)=H_{j k}^{* \lambda} \frac{d x^{j}}{d s} \frac{d x^{k}}{d s},
$$

par conséquent, le vecteur $B_{i}{ }^{\lambda} \frac{d x^{i}}{d \delta}$ ne se déplace parallèlément que dans le cas oû nous avons $H_{j k}^{\cdot \lambda} \frac{d x^{j}}{d s} \frac{d x^{k}}{d s}=\mathbf{0}$.

Or, nous allons considérer un vecteur $V^{\lambda}$ de $E_{m}$ qui se déplace toujours parallèlement à lui-même dans la direction déterminée par $v^{i}=B_{{ }_{\cdot} \lambda}^{i} V^{\lambda}$. La direction $v^{2}$ dans $V_{n}$ décrit une courbe. C'est la ligne la plus droite relativement à $E_{m}$ considérée par MM. Einstein et Mayer pour représenter la trajectoire d'une particule dans le champ gravifique et éléctromagnétique.

Posons

$$
\frac{d x^{i}}{d r}=B_{\cdot \lambda}^{i} V^{2}
$$

Alors, on trouve

$$
V^{\lambda}=B_{i}^{\cdot \lambda} \frac{d x^{i}}{d i^{*}}+B_{P}^{\cdot \lambda} v^{P},
$$

où $v^{P}$ est défini par

$$
v^{P}=B_{\cdot{ }_{\lambda}}^{P} V^{\lambda} .
$$

Or, d'après la définition, on a, de (6.3), 


$$
\begin{aligned}
\frac{\delta}{d r} V^{\lambda} & =B_{i}^{\cdot \lambda}\left(\frac{\delta^{2} x^{i}}{d r^{2}}-H_{\cdot k P}^{i} v^{P} \frac{d x^{k}}{d r}\right)+B_{P}^{\bullet^{\lambda}}\left(H_{j k}^{* P} \frac{d x^{j}}{d r} \frac{d x^{k}}{d r}+\frac{\delta}{d r} v^{P}\right) \\
& =\alpha V^{\lambda}=a\left(B_{i}^{\cdot \lambda} \frac{d x^{i}}{d r}+B_{P^{\lambda}}^{{ }^{\lambda}} v^{P}\right),
\end{aligned}
$$

d'où

$$
\frac{\delta^{2} x^{i}}{d r^{2}}+H_{\cdot k P}^{i} v^{P} \frac{d x^{j^{2}}}{d r}=\alpha \frac{d x^{i}}{d r}
$$

et

$$
\frac{\delta}{d r} v^{P}+H_{j k}^{* P} \frac{d x^{j}}{d r} \frac{d x^{k}}{d r}=\alpha v^{P} .
$$

Si l'on suppose que $V^{\lambda}$ soit un vecteur unitaire, on aura $\alpha=0$.

§7. $V_{n}$ plan ou géodésique relativement $\grave{a} E_{m}$.

Si l'espace vectoriel linéaire $E_{n}$ se déplace parallèlement à lui-même 'quand on déplace dans'n'importe quelle direction, on diț que $V_{n}$ est plan relativement à $\boldsymbol{E}_{m}$.

Pour cela, on doit avoir

$$
B_{\bullet \lambda}^{P} \delta\left(B_{j}^{\cdot \lambda} v^{j}\right)=H_{j k}^{{ }^{P}} v^{j} d x^{k}=0,
$$

pour n'importe quel vecteur $v^{i}$ et pour n'imorte quelle/direction $d x^{i}$, donc,

$$
H_{j k}^{* P}=0 \quad \text { ou } \quad H_{j_{k}}^{\ddot{\lambda}^{\lambda}}=\mathbf{0} \text {. }
$$

Dans ce cas, l'espace vectoriel linéaire $E_{m-n}$ se déplace aussi parallèlement à lui-même, comme on peut du reste le vérifier en tenant compte de (2.15).

Si le vecteur $B_{i}^{\cdot \lambda} \frac{d x^{i}}{d s}$ de $E_{m}$ tangent à une géodésique de $V_{n}$ se déplace toujours parallèlement à lui-même le long de la géodésique, on dit que $V_{n}$ est géodésique relativement à $\boldsymbol{E}_{m}$.

Pour cela, on doit avoir

$$
\frac{\delta}{d s}\left(\dot{B}_{i}^{\circ \lambda} \frac{d x^{i}}{d s}\right)=H_{j k}^{\cdot \lambda} \frac{d x^{j}}{d s} \frac{d x^{k}}{d s}=0
$$

pour n'importe quelle direction $\frac{d x^{j}}{d s}$, d'où

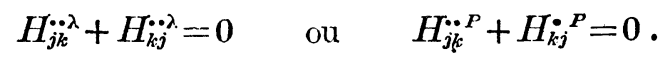

\$8. La théorie d'Einstein et Mayer et la généralisation par Michal el Botsford.

Dans leur théorie unitaire des champs, MM. Einstein et Mayer ont considere un $V_{4}$ géodésique ralativement à $E_{5}$. Examinons ce cas, en supposant que $V_{n}$ soit géodésique relativement à $E_{m}$.

Dans ce cas, les équations de la ligne la plus droite relativement à $E_{m}$ s'écri- 
vent

$$
\frac{d^{2} x^{i}}{d s^{2}}+\left\{\begin{array}{l}
i \\
j k
\end{array}\right\} \frac{d x^{j}}{d s} \frac{d x^{k}}{d s}=H_{\bullet k P}^{i} v^{P} \frac{d x^{k}}{d s}, \frac{\delta}{d s} v^{P}=0
$$

donc, $v^{P}$ se déplace parallèlement d'après la connexion de $\boldsymbol{E}_{m-n}$.

Or, l'équation (5.5) s'écrit dans ce cas

$$
K_{\mu k}=B_{\bullet \mu}^{j}\left(R_{j k}+H_{j a}^{{ }^{P}} H_{\bullet k P}^{a}\right)-B_{\bullet \mu}^{Q} H_{\bullet k Q ; a}^{a},
$$

et l'équation (5.7)

$$
K=R+H_{\bullet i}^{a{ }^{P}} H_{\bullet a P}^{b} \cdot
$$

Posons, avec Einstein et Mayer,

$$
\begin{aligned}
& U_{\mu k}=K_{\mu k}-\frac{1}{4} B_{k \mu}(K+R) \\
& =B_{\bullet \mu}^{j}\left(R_{j k}-\frac{1}{2} R g_{j k}+H_{j a}^{\ddot{p}^{P}} H_{\bullet k P}^{a}-\frac{1}{4} H_{\bullet b}^{a{ }^{P}} H_{\bullet a}^{v}{ }^{P} g_{j k}\right) \\
& -B_{\cdot \mu}^{Q} H_{\bullet k Q ; a}^{a} \cdot
\end{aligned}
$$

En dérivant (8.4) covariantement, on trouve

$$
\begin{aligned}
& U_{\mu k ; h}=H_{\bullet h \mu}^{j}\left(R_{j k}-\frac{1}{2} R g_{j k}+H_{j a}^{{ }^{P}} H_{\bullet k P}^{a}-\frac{1}{4} H_{\bullet b}^{a{ }^{P}} H_{\bullet a P}^{u} g_{j k}\right) \\
& +B_{\bullet \mu}^{j}\left[R_{j k ; h}-\frac{1}{2} R_{; h} g_{j k}+H_{j_{a ; h}}^{. p} H_{\bullet k P}^{a}+H_{\dot{j a}^{P}} H_{\bullet k P ; h}^{a}\right. \\
& \left.\left.-\frac{1}{4} H_{\bullet b ; h}^{a \cdot P} H_{\bullet a P}^{b}+H_{\bullet b}^{a \cdot P} H_{\bullet a P ; h}^{b}\right) g_{j k}\right] \\
& +B_{i \mu} H_{\bullet h}^{i} H_{* k Q ; a}^{a}-B_{* \mu}^{Q} H_{* k Q ; a ; h}^{a},
\end{aligned}
$$

d'où

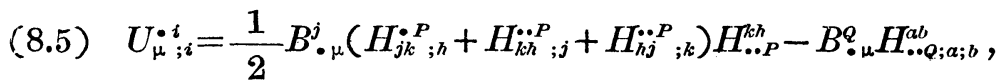

grâce à l'identité (4.5) et $R_{j k}=R_{k j}$.

D'autre part, on a

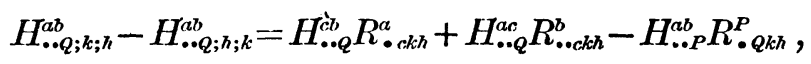

d'où, en posant $a=k, b=h$,

$$
2 H_{\bullet Q ; a ; b}^{a b}=-H_{\cdot \bullet P}^{a b} R_{\bullet Q a b}^{P} \text {. }
$$

En substituant (8.6) dans (8.5), on obtient finalement

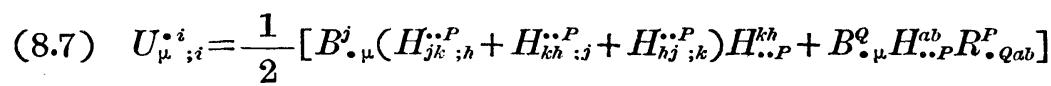

Examinons le cas où $n=m-1$. Dans ce cas, en posant

$$
B_{i n}^{: \lambda}=B^{\lambda}, \quad H_{j k}^{: \dot{n}}=H_{j k}, \quad \text { etc. }
$$

nous avons, de (8.4),

$$
U_{\mu k}=B_{\bullet \mu}^{j}\left[\left(R_{j k}-\frac{1}{2} R g_{j k}\right)+\left(H_{j a} H_{\bullet \cdot k}^{a}-\frac{1}{4} H_{\bullet b}^{a} H_{\bullet a}^{b} g_{j k}\right)\right]-B_{\mu} H_{\bullet k ; a}^{a},
$$


et de (8.7)

$$
U_{\mu ; i}^{\bullet i}=\frac{1}{2} B_{\cdot \mu}^{j}\left(H_{j k ; h}+H_{k h ; j}+H_{h j ; k}\right) H^{k h},
$$

parce que $R_{\bullet Q k h}^{P}=0$.

Ceux qui correspondent aux équations des champs d'Einstein et Mayer sont

$$
U_{\mu k}=0 \quad \text { et } \quad H_{j k ; h}+H_{k h ; j}+H_{h j ; k}=0 \text {, }
$$

d'où on a

$$
\text { (8.9) } \quad\left(R_{j k}-\frac{1}{2} R g_{j k}\right)+\left(H_{j a}^{\cdot} H_{\bullet k}^{a}-\frac{1}{4} H_{\bullet b}^{a} H_{\bullet a}^{b} g_{j k}\right)=0,
$$

et

$$
H_{\cdot k ; a}^{a}=0
$$

$H_{j k}$ étant de la forme

$$
H_{j k}=\frac{\partial \varphi_{j}}{\partial x^{k}}-\frac{\partial \varphi_{k}}{\partial x^{j}}
$$

où $\varphi_{j}$ est un vecteur covariant qui correspond au potentiel éléctomagnétique. 